\title{
Autonomic dysreflexia in a case of radiation myelopathy and cisplatin-induced polyneuropathy
}

\author{
Hiroshi Saito ${ }^{1}$
}

Received: 6 June 2020 / Revised: 29 July 2020 / Accepted: 29 July 2020

(c) International Spinal Cord Society 2020

\begin{abstract}
Introduction While autonomic dysreflexia caused by severe spinal cord lesions can be life-threatening, relevant reports on non-traumatic spinal lesions are rare. Furthermore, modes of innervation of the supraspinal inhibitory pathways at each spinal sympathetic segment remain unknown. Herein, I report the case of a patient with autonomic dysreflexia and radiation myelopathy. The laterality of autonomic dysreflexia was investigated with special reference to the sudomotor function.

Case presentation A 51-year-old man with a history of epipharynx carcinoma, radiotherapy, and cisplatin chemotherapy was referred for the evaluation of autonomic function. He was ambulant but displayed spastic tetraparesis, areflexia of the extremities, sensory disturbance below $\mathrm{C} 4$ dermatome, dysuria, and impotence. Spinal magnetic resonance imaging demonstrated a cervical lesion involving the lateral portion of $\mathrm{C} 2-\mathrm{C} 5$, bilaterally. The thermal sweating test showed that sweating was lower on the left side of the face and neck, left shoulder, and arm than the corresponding parts on the right side. The rest of the body was anhidrotic. Sweating due to autonomic dysreflexia was symmetric, but more abundant on the left side of the face. Acetylcholine-induced sweating was markedly reduced on the left leg.

Discussion This might be the first documentation of autonomic dysreflexia observed in a patient with radiation myelopathy. The present observations suggested that the supraspinal inhibitory pathway to spinal preganglionic neurons may descend on the same side as thermal sudomotor facilitatory pathways at the cervical level. Furthermore, autonomic dysreflexia was more prominent in the standing position suggesting that the pressure stimulus might enhance autonomic dysreflexia.
\end{abstract}

\section{Introduction}

Severe spinal cord lesion may disrupt autonomic nervous system functions, such as cardiovascular control and sweating. Generally, the skin area innervated by segments below the lesions may show thermal hypo- or anhidrosis [1], though rare cases may display continuous hyperhidrosis [2]. Conversely, excessive sweating may be provoked by various sensory inputs to the disconnected spinal segments, a condition termed as autonomic dysreflexia (AD). Head and Riddoch [3] demonstrated that, while the bladder distension might be the most powerful triggering factor of $\mathrm{AD}$, other endogenous and exogenous stimuli may also cause AD. This reflex sweating together with an abrupt increase in

Hiroshi Saito

saito-tubame@jcom.home.ne.jp

1 Department of Neurology, Sendai Eastern Neurosurgical Hospital, 1-12-1 Iwakiri, Sendai 983-0821, Japan blood pressure (BP) are regarded as a part of mass reflex, being a released phenomenon from supraspinal tonic inhibition originated in the medulla [4-6]. When the lesion is located at the cervical cord, the whole body may be thermally anhidrotic, and AD-sweating may occur all over the body, being more prominent on the upper part of the body $[1,7]$.

Recent animal and human studies have suggested that various functional and morphological changes may occur in the spinal cord, at the lesion site and also above and below the lesion level. These alterations include morphological changes of the sympathetic preganglionic neurons, interneurons damage, proliferation of the afferent nerve terminals including those of large myelinated proprioceptive afferents, maladaptive plasticity of the neuronal circuits, and altered neurotransmitter balance, all of which may contribute to clinical features and varying degrees of AD [811]. To date, the innervation modes of the supraspinal inhibitory pathways with respect to each level of sympathetic segment and laterality remain unsettled. 
Fig. 1 MRI images of the cervical cord. Gadoliniumenhanced T1-weighted magnetic resonance images of the cervical cord showing bilaterally involved lateral corticospinal and reticulospinal tracts.
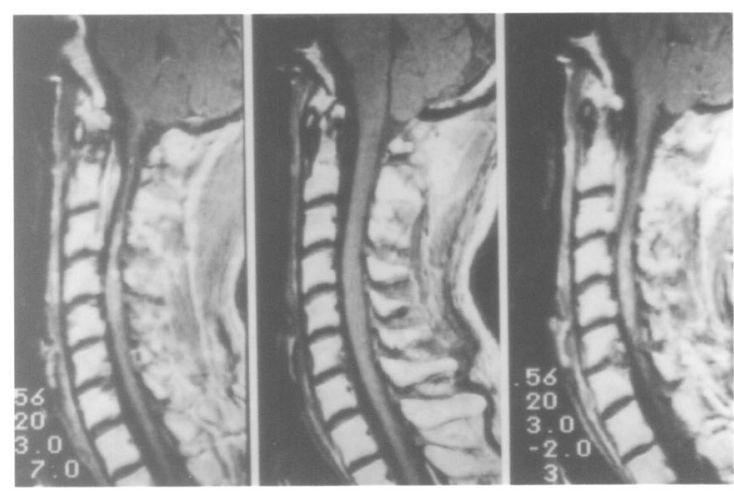

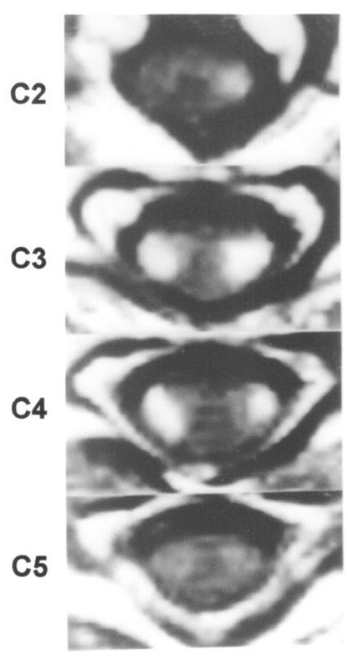

Reports on AD due to the non-traumatic spinal lesion are rare [12-17]. Herein, I report the case of a patient with radiation myelopathy and cisplatin-induced neuropathy presented $\mathrm{AD}$ together with the results of qualitative and quantitative sweat tests. The present observations suggested following two points; (1) the supraspinal inhibitory pathway to spinal preganglionic neurons may descend on the same side as the thermal sudomotor facilitatory pathways being involved in/near the lateral reticulospinal tract at the cervical level, and (2) AD was more prominent in the standing position, suggesting that the pressure stimulus might enhance the AD.

\section{Case presentation}

A 51-year-old man was admitted to our hospital because of progressive gait disturbance, weakness of the left upper limb, and dysuria. At 2 years before the present admission, he received radiation therapy $(63 \mathrm{~Gy})$ and cisplatin chemotherapy (total $220 \mathrm{mg}$ ) for epipharynx carcinoma. Five months later, he developed difficulty in walking, followed by odynophagia, for which tracheostomy was performed after the diagnosis of radiation chondritis. At 1 year before the present admission, he noted weakness and numbness of the left limbs which extended to the opposite side and was followed by micturition difficulties. He noted that he perspired a lot on the face, neck, and on the shoulder, whenever he had the desire to void.

Physically, he was alert, afebrile, and ambulant, though he had a tracheal cannula, and required intermittent selfcatheterization. The $\mathrm{BP}$ and heart rate (HR) were 95/ $60 \mathrm{mmHg}$ and 60 beats per $\mathrm{min}(\mathrm{bpm})$ and 90/62 $\mathrm{mmHg}$ and $78 \mathrm{bpm}$, in supine and standing positions, respectively. The cranial nerves were intact. He showed tetraparesis with spasticity more prominent on the left than the right side. On bedside inspection, fasciculations were observed in muscles innervated by C5-C7 cervical segments. Deep tendon reflexes were absent in the lower extremities. Babinski's sign was positive on the left. He had hypoesthesia below $\mathrm{C} 4$ dermatome on both sides. The deep sensation was diminished on the both lower limbs.

Laboratory data were unremarkable. Cerebrospinal fluid cell count was 2 cells $/ \mathrm{mm}^{3}$. Protein content was $26 \mathrm{mg} / \mathrm{dL}$. Magnetic resonance imaging of the cervical cord revealed that C5-C7 segments were swollen and showed high signal intensity on T2-weighted images. On T1-weighted images, the lesion was slightly hypointense and was enhanced by Gadolinium-EDTA. The Axial sections showed bilaterally enhanced lesions mainly involving the lateral portion (Fig. 1).

\section{Methods of investigation of sudomotor function}

After obtaining an informed consent from the patient and his family, the following sweat tests were performed. Thermal sweating (TS) was induced by heating the patient in the supine position with electric blankets. Deep forehead temperature was monitored by a deep-body thermometer (Core-temo-CNT-204, Probe-No-ME-PD-51, TerumoJapan) that can detect temperature to a depth up to $15 \mathrm{~mm}$. TS was evaluated qualitatively using the modified Minor's colorimetric method [18], and the sweat rate was monitored with a capacitance hygrometer (Hydrograph-AMU-2, Kyokuto-Denshi, Nagoya, Japan) placed on the forehead, the volar aspect of the forearm, and the lateral aspect of the leg. To evaluate the postganglionic sympathetic function, acetylcholine-induced sweating (Ach-S) was achieved by injecting $0.05 \mathrm{ml}$ of $5 \%$ acetylcholine chloride on the forearm and the leg on the left side. 
Fig. 2 Qualitative sudomotor evaluation using a modified Minor's colorimetric test. a Thermal sweating shows discoloration restricted to the face and right side of the neck and right shoulder. b Sweating area due to autonomic dysreflexia elicited by the bladder distension is almost symmetric on the upper part of the body. (Areas below T4 and distal parts of upper limbs are not covered by test papers). Numerals below the figures correspond to the final deepbody temperatures on the forehead/time in min after the start of heating.

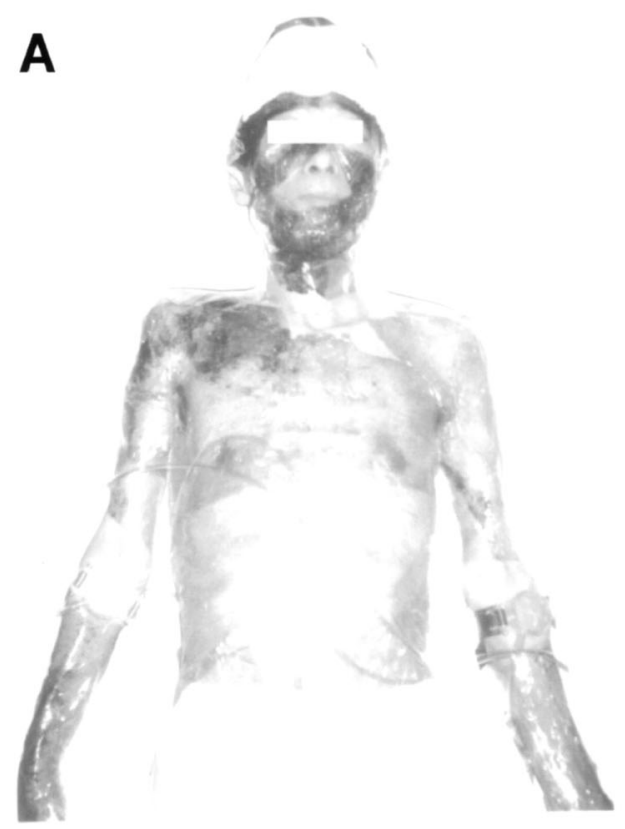

B

$37.8 / 60$

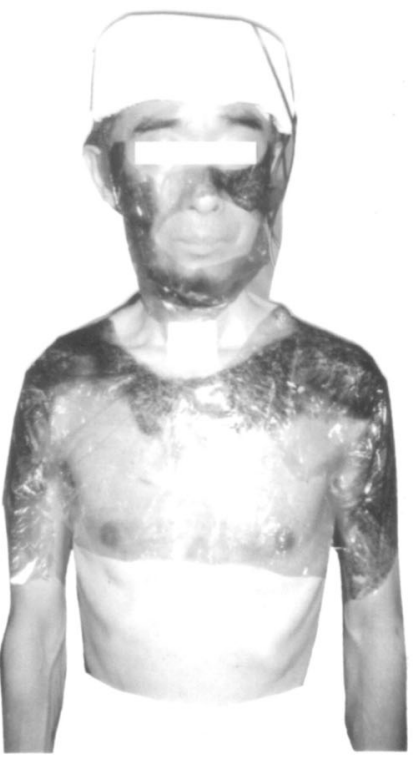

$36.4 / 30$

\section{A}
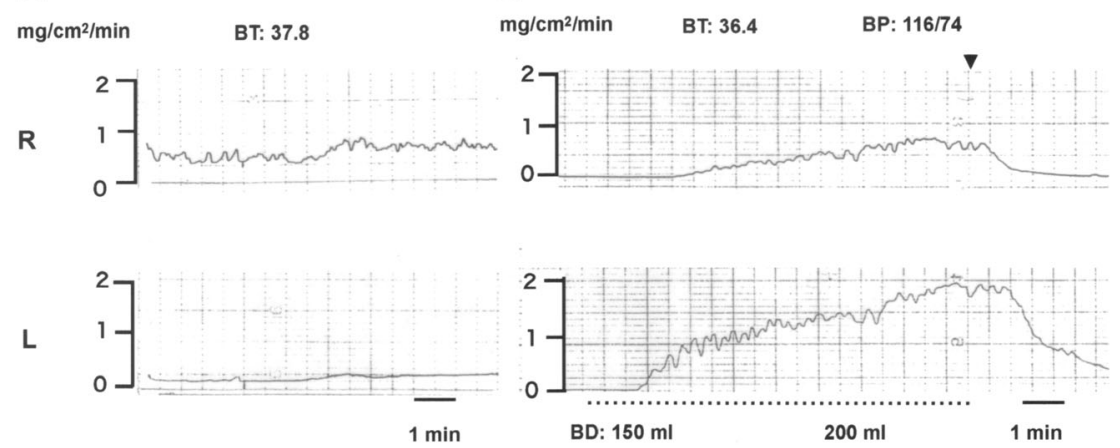

Fig. 3 Sweat rates on the forehead. Sweat rates on the forehead during thermal sweating at core temperature $37.8^{\circ} \mathrm{C}$ (a) and sweating due to autonomic dysreflexia at room temperature (b). On the thermal sweating test, the sweat rates were lower on the left side than the right

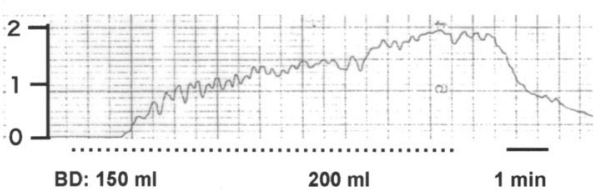

side of the face, whereas sweating due to autonomic dysreflexia was apparently abundant on the left. The dotted line and the arrowhead represent the bladder distension and evacuation, respectively.

TS was largely defective. Before heating the patient showed no sweating. Fifty minutes after the heating start (core temperature: $37.8^{\circ} \mathrm{C}$ ), discoloration was noted on the right side of the forehead, neck, and around the right shoulder involving $\mathrm{T} 1 \mathrm{sensory}$ dermatome. The right shoulder and the upper limb showed patchy sweat spots. On the left side, the face showed only slight discoloration. The rest of the body was anhidrotic (Fig. 2a). The sweat rates $\left(\mathrm{mg} / \mathrm{cm}^{2} / \mathrm{min}\right)$ on the forehead were 0.578 and 0.218 on the right and left sides, respectively (Fig. 3a). Those on both forearms and the left upper chest (T2) were null, and 0.062 on the right side of the upper chest. The Ach-S rate was normal on the left forearm (0.525), but was markedly reduced on the left leg (0.034) compared with the age- and sex-matched participants without neurological or autonomic deficits $(0.362 \pm 0.155$ on the forearm and $0.354 \pm 0.198$ on the leg $[n=11])$.

Sweating due to AD (AD-S) was induced naturally; after having withheld the self-catheterization for several hours, the patient lied on the bed with his face and upper parts of the body wrapped with test sheets. When he had the desire to void, no sweating was observed. Following this, he was requested to stand up and walk around. In a few minutes, his desire to void became intense, and sweating appeared rapidly on the face and neck, and then on the upper chest (Fig. 2b). After he performed self-catheterization, sweating 
subsided almost immediately. A similar evaluation was done during the urodynamic study. The baseline BP and HR before the catheter insertion were $92 / 60 \mathrm{mmHg}$ and $64 \mathrm{bpm}$, respectively. No apparent sweating was detected during and after the insertion of the indwelling tube. BP and HR were counted at $103 / 66 \mathrm{mmHg}$ and $57 \mathrm{bpm}$, respectively. After $150 \mathrm{ml}$ of saline was infused, the patient developed a desire to void. Sweating appeared on the left side of the face and the upper part of the body, followed by sweating on the right side. After increasing the volume of the infused saline to $200 \mathrm{ml}$, he had an intense sensation of the bladder filling. $\mathrm{BP}$ and HR increased slightly to $116 / 74 \mathrm{mmHg}$ and $67 \mathrm{bpm}$, respectively. The infusion was stopped, and the bladder was evacuated. Sweating subsided within a few minutes, and BP and HR returned to the pre-distension levels. The maximum sweat rate on the forehead was 0.779 and 1.807 on the right and left sides, respectively (Fig. 3b). During bladder distension, the patient had no headache or chill but reported a vague dull sensation in the lower abdomen. The urological evaluation revealed a normal upper urinary tract, uninhibited bladder with a maximum volume of $200 \mathrm{ml}$. At the distension with over $150 \mathrm{ml}$ saline, detrusor sphincter dyssynergia was observed.

\section{Discussion}

Our patient was diagnosed with radiation myelopathy and cisplatin-induced polyneuropathy based on the history and radiological findings $[19,20]$. Investigations of the patient's autonomic functions revealed several important features of the pathophysiological mechanisms of AD. Radiological findings and comparison of TS and AD-sweating (AD-S) suggested that both of the sudomotor-related hypothalamospinal facilitatory pathways and the supraspinal inhibitory pathways may descend in the lateral portion of the cervical cord, ipsilaterally.

Previously, we reported two cases of the lateralized AD-S. The first case was due to an incomplete cervical lesion caused by a ruptured cervical arteriovenous malformation [12], and the second was of lateral medullary infarction with dysuria [13]. In those patients, TS was reduced, and AD-S was more abundant on the lesion side. Similarly, in the present case, AD-S was more abundant on the side where TS was more defective. Neuroanatomical animal studies have suggested that the brainstem projections to the intermediolateral cell column are mainly ipsilateral $[4,21]$.

In the present case, whereas the bladder distension alone provoked AD-S as observed during the urodynamic study, standing and walking appeared to have acted as an additional triggering factor of AD. According to Guttmann [1], postural changes influence AD-S, especially in patients with cervical lesions. In cases reported by List and Pimenta [7], AD-S was exaggerated in the sitting position and by frequent spasms of the lower extremities. Suhaida and Engkasan [22] reported an interesting case of a patient with traumatic C6 impairment and excessive sweating on the right side of the face which only occurred when lying in the supine or left lateral positions.

In normal physiological conditions, the pressure stimuli applied to one side of the body inhibits TS on that side and augments it on the opposite side [23, 24]. In the supine position, pressing the skin of the back bilaterally inhibits sweating on the upper part of the body. Conversely, while standing or sitting erect, the pressure stimulus on the lumbosacral segments enhances and inhibits sweating on the rostral and lower parts of the body, respectively [24].

Our observations suggested that non-painful proprioceptive inputs from pressure stimuli induced due to postural changes might have exacerbated the autonomic response under the disinhibited condition [1]. Animal studies have suggested that after a spinal cord injury, the large-diameter afferent fibers show marked sprouting at the level of the injury [25]. Moreover, activation of large-diameter somatic afferents triggers the increase in vasoconstrictor drive that leads to AD.

Recent animal studies have increased our understanding concerning the pathophysiological mechanisms of $\mathrm{AD}$, especially those of cardiovascular disorders. However, several questions remain unanswered such as (1) the reasons why $\mathrm{AD}$ does not develop in patients with spinal lesions below T10 segments [1] and (2) the mode of innervation of the supraspinal inhibitory pathways to each sympathetic segment of the spinal cord. Sudomotor function is uniquely developed in humans, and excessive sweating is one of the common features of $\mathrm{AD}$. Investigations on whole body sudomotor function in patients with $\mathrm{AD}$ might help to address the aforementioned questions regarding $\mathrm{AD}[1,26]$.

\section{Data availability}

All data generated or analyzed during this study are included in this published article.

Acknowledgements The author would like to thank late Dr. Tetsutaro Ohnuma, Department of Urology, Tohoku University, Sendai, Japan for performing urodynamic studies, and to Editage (www.editage.com) for English language editing.

\section{Compliance with ethical standards}

Conflict of interest The author declares no conflict of interest.

Publisher's note Springer Nature remains neutral with regard to jurisdictional claims in published maps and institutional affiliations. 


\section{References}

1. Guttmann L. Spinal Cord Injuries. Comprehensive management and research. Oxford: Blackwell Scientific Publications; 1973.

2. Kneisley LW. Hyperhidrosis in paraplegia. Arch Neurol. 1977;34:536-9.

3. Head H, Riddoch G. The autonomic bladder, excessive sweating and some other reflex conditions, in gross injuries of the spinal cord. Brain. 1917;40:188-263.

4. Amendt K, Czachurski J, Dembowsky K, Seller H. Bulbospinal projection to the intermediolateral cell column; a neuroanatomical study. J Auton Nerv Syst. 1979;1:103-7.

5. Dembowsky K, Czachurski J, Amendt K, Seller H. Tonic descending inhibition of the spinal somato-sympathetic reflex from the lower brain stem. J Auton Nerv Syst. 1980;2:157-82.

6. Karlsson AK. Autonomic dysreflexia. Spinal Cord. 1999;37:383-91.

7. List CF, Pimenta AD. Sweat secretion in man. VI. Spinal reflex sweating. Arch NeuroPsychiatry. 1944;51:501-7.

8. Rabchevsky AG. Segmental organization of spinal reflexes mediating autonomic dysreflexia after spinal cord injury. Prog Brain Res. 2006;152:265-74.

9. Eldahan KC, Rabchevsky AG. Autonomic dysreflexia after spinal cord injury: systemic pathophysiology and methods of management. Auton Neurosci. 2018;209:59-70.

10. Shouman K, Benarroch EE. Segmental spinal sympathetic machinery: implications for autonomic dysreflexia. Neurology. 2019;93:339-45.

11. Michael FM, Patel SP, Rabchevsky AG. Intraspinal plasticity associated with the development of autonomic dysreflexia after complete spinal cord injury. Front Cell Neurosci. 2019;13:505.

12. Tokudome T, Saito $H$. Unilateral spinal sweating observed in a case of incomplete high cervical cord lesion. Neurol Med. 1985;22:61-67. (In Japanese with English abstract)

13. Saito H. Ipsilateral hyperreflexic sweating in a case of lateral medullary infarction with dysuria. Auton Nerv Syst. 1986;23:417-20. (In Japanese with English abstract)
14. Sakakibara R, et al. Autonomic dysreflexia due to neurogenic bladder dysfunction; an unusual presentation of spinal cord sarcoidosis. J Neurol Neurosurg Psychiatry. 2001;71:819-20.

15. Kulcu DG, Akbas B, Citci B, Cihangiroglu M. Autonomic dysreflexia in a man with multiple sclerosis. J Spinal Cord Med. 2009;32:198-203.

16. Jayakrishnan MP, Krishnakumar P, Gauthamen R, Sabitha S, Devarajan E. Autonomic dysreflexia in acute disseminated encephalomyelitis. Pediatr Neurol. 2012;47:309-11.

17. Furlan JC. Autonomic dysreflexia following acute myelitis due to neuromyelitis optica. Mult Scler Relat Disord. 2018;23:1-3.

18. Saito H, Kobayashi K, Mochizuki H, Ishii T. Axonal degeneration of the peripheral nerves and postganglionic anhidrosis in a patient with multiple sclerosis. Tohoku J Exp Med. 1990;162:279-91.

19. Khan M, et al. Radiation-induced myelitis: Initial and follow-up MRI and clinical features at a single tertiary care institution during 20 years. AJNR Am J Neuroradiol. 2018;39:1576-81.

20. Avan A, et al. Platinum-induced neurotoxicity and preventive strategies: past, present, and future. Oncologist. 2015;20:411-32.

21. Tohyama M, Sakai K, Touret M, Salvert D, Jouvet M. Spinal projections from the lower brain stem in the cat as demonstrated by the horseradish peroxidase technique. II. Projections from the dorsolateral pontine tegmentum and raphe nuclei. Brain Res. 1979;176:215-31.

22. Suhaida SS, Engkasan JP. A report on an unusual presentation of autonomic dysreflexia. Med J Malays. 2012;67:616-7.

23. Takagi K, Sakurai T. A sweat reflex due to pressure on the body surface. Jpn J Physiol. 1950;1:22-28.

24. Kuno Y. Human perspiration. Springfield: Charles C Tomas Publisher; 1956.

25. Brown R, Burton AR, Macefield VG. Autonomic dysreflexia: somatosympathetic and viscerosympathetic vasoconstrictor responses to innocuous and noxious sensory stimulation below lesion in human spinal cord injury. Auton Neurosci. 2018;209:71-78.

26. Saito $\mathrm{H}$. The location and characteristics of the thermal sudomotor pathways in the human brainstem: a reappraisal. Auton Neurosci. 2019;217:80-90. 